\title{
Morphological and functional MDCT: problem- solving tool and surrogate biomarker for hepatic disease clinical care and drug discovery in the era of personalized medicine
}

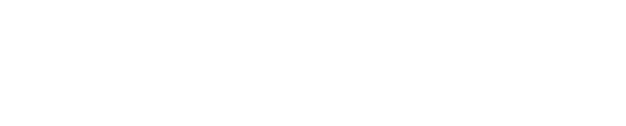

Liang Wang

Department of Radiology, Tongji Hospital, Tongji Medical College, Huazhong University of Science and Technology, Wuhan, PR China
Correspondence: Liang Wang Department of Radiology, Tongii Hospital, Tongji Medical College, Huazhong University of Science and Technology, 1095 Jiefang Rd, Wuhan 430030, PR China

$\mathrm{Tel}+|-2| 2-639-22 \mid 4$

Email wang6@mskcc.org

\begin{abstract}
This article explains the significant role of morphological and functional multidetector computer tomography (MDCT) in combination with imaging postprocessing algorithms served as a problem-solving tool and noninvasive surrogate biomarker to effectively improve hepatic diseases characterization, detection, tumor staging and prognosis, therapy response assessment, and novel drug discovery programs, partial liver resection and transplantation, and MDCTguided interventions in the era of personalized medicine. State-of-the-art MDCT depicts and quantifies hepatic disease over conventional CT for not only depicting lesion location, size, and extent but also detecting changes in tumor biologic behavior caused by therapy or tumor progression before morphologic changes. Color-encoded parameter display provides important functional information on blood flow, permeability, leakage space, and blood volume. Together with other relevant biomarkers and genomics, the imaging modality is being developed and validated as a biomarker to early response to novel, targeted anti-VEGF(R)/PDGFR or antivascular/ angiogenesis agents as its parameters correlate with immunohistochemical surrogates of tumor angiogenesis and molecular features of malignancies. MDCT holds incremental value to World Health Organization response criteria and Response Evaluation Criteria in Solid Tumors in liver disease management. MDCT volumetric measurement of future remnant liver is the most important factor influencing the outcome of patients who underwent partial liver resection and transplantation. MDCT-guided interventional methods deliver personalized therapies locally in the human body. MDCT will hold more scientific impact when it is fused with other imaging probes to yield comprehensive information regarding changes in liver disease at different levels (anatomic, metabolic, molecular, histologic, and other levels).
\end{abstract}

Keywords: perfusion MDCT, liver nodule, angiogenesis, functional imaging, VEGF receptor

Personalized medicine means tailoring medical care to ensure the delivery of the right therapy for the right patient at the right time based on a patient's unique genetic blueprint. ${ }^{1-3}$ Personalized medicine based on the cutting-edge technology is already useful in saving patients' lives and reducing healthcare costs and would radically reform the manner of traditional medical practice. Together with other relevant biomarkers and genomics, radiology techniques and imaging methods are the problem-solving tools to help guide treatment to meet the growing demand for patient-individualized treatment that can minimize treatment morbidity while maximizing treatment benefits. ${ }^{4,5}$ Current innovations in morphological and functional multidetector computer tomography (MDCT) in combination with advance imaging postprocessing algorithms are revolutionizing the traditional interpretation and analysis of radiology examination, 
and are being developed and validated as a problem-solving tool and surrogate biomarker for hepatic disease characterization, detection, tumor staging, prognosis, therapy response assessment, novel drug discovery programs, and partial liver resection and transplantation, and MDCT-guided interventions, thus impacting upon personalized medicine..$^{6-8}$

\section{Unique liver anatomy and kinetics}

Analysis of contrast enhancement of the liver is more complex for the following reasons: (1) the sinusoids instead of the capillary, (2) the sinusoidal endothelium (no basement membrane) instead of the capillary endothelium, (3) the space of Disse (or perisinusoidal space) instead of the extravascular extracellular compartment, (4) two blood inflow components in two different time settings to the liver (the hepatic artery and the portal vein), and (5) more challenging due to respiratory motion.

At the least, 4 different vascular phases of the liver that should be included are the precontrast, arterial, portal (or equilibrium), and delayed (or venous) phases. ${ }^{9}$ Hepatic arterial phase occurs at 20-30 seconds post injection (with contrast medium), which provides optimum contrast to the hepatic artery and its branches. Approximately 30\% of liver lesions are detectable exclusively on hepatic arterial phase images. ${ }^{10}$ Hepatic hypervascular lesions reach peak enhancement and are best seen in hepatic arterial phase. ${ }^{11,12}$ Hepatic portal phase is usually acquired at 30-35 seconds post injection (with contrast medium). Hepatic venous phase at approximately 60 seconds post-injection (with contrast medium), when the contrast material has arrived at the hepatic veins, and hepatic delayed phase 120-180 seconds post-injection (with contrast medium), when contrastmaterial equilibrium of the intravascular and extravascular components occurs. ${ }^{12}$ The timing bolus technique and adjustment of the total amount of contrast according to the body weight will compensate for differences in cardiac output and distribution volume.

\section{State-of-the-art MDCT and new-generation iodinated contrast media}

With the introduction of modern, fast, dual-source volumetric cine scanners, state-of-the-art MDCT data acquisition has become more robust for qualitative and quantitative analysis. ${ }^{13-16}$ The advantages of MDCT (64 slices) includes the following: (1) improved spatial/longitudinal resolution $(0.4 \mathrm{~mm})$ and increased $z$-axis coverage $(80 \mathrm{~mm})$ using submillimeter collimation, providing an isotropic data set, and reducing partial-volume effects; ${ }^{17,18}$ (2) shortening of scan time (0.33-second rotation speed of the gantry, $<4$ seconds for the entire liver) allowing short breath-holding periods, which reduces motion artifacts and provides sharper distinction of each vascular phase; ${ }^{19,20}$ (3) improved 3D reconstructions for clarification of interfaces predominantly situated in the $z$-axis, providing clear/detailed distinction of liver lesion ${ }^{12}$; (4) the ability to apply a multitude of imagingpostprocessing techniques..$^{20,21}$

Potential biological hazards of radiation associated with MDCT must be considered in achieving both high-temporal resolution and high-spatial resolution imaging of the entire liver. Radiation exposure can be kept under control by using a short scanning time, reduced tube current and tube potential, automatic device-modulating dose delivery in conformity to human body, different X-ray exposure in repeated volume acquisitions. ${ }^{19,22}$ Currently, commercially available software packages to monitor radiation dosage include WinDose; Wellhofer Dosimetry, Schwarzenbruck, Germany; a cylindrical phantom (Radiation Monitor Controller, model 9015; Radocal Corp, Monrovia, California, USA). To reduce potential biological hazards of radiation, the protocol parameters recommended in hepatic MDCT are slice collimation of $4 \times 5 \mathrm{~mm}$, $80-120 \mathrm{kV}, 100-200 \mathrm{~mA}$, temporal solution is 4-5 seconds per scan, and weighted CT dose index of $4.16 \mathrm{mGy}^{22,23}$

The risk of adverse reaction or renal toxicity of new-generation iodinated contrast media used in MDCT is quite low. ${ }^{24}$ Moreover, macromolecular contrast media, such as liposomes containing nonionic iodinated contrast media or gold nanoparticles, has longer intravascular half-life and may improve for imaging angiogenesis and perfusion quantification. ${ }^{25-28}$

\section{Advanced computational image analysis techniques}

Advanced computational image analysis techniques have been developed not only to detect liver lesions but also to quantitatively measure liver lesions. ${ }^{29-32}$ As performed more objectively, reproducibly, and efficiently, especially when attempting to measure volumetrically, the computerized quantitative measurements may ultimately replace manual measurements. ${ }^{33}$ The clinical application of computational image analysis techniques has substantial implications for monitoring tumor therapy in an individual patient and for evaluating the effectiveness of new therapies under development. . $9,30,33,34^{2}$

Various imaging-postprocessing algorithms have been developed to (semi)automatically segment liver contour and liver lesions on MDCT. ${ }^{30,33,35,36}$ For instance, snake algorithm with gradient vector flow implements liver segmentation. 
Shape-constraint region-growing algorithm facilitates automatic delineation of liver lesions and provides unidimension (the largest diameter of the lesion on the transverse image containing the largest cross-sectional lesion area), bidimension (the product of the largest diameter and the largest perpendicular diameter on the same transverse image), and the area on the transverse image for each of the segmented lesions (Figure 1).

Currently, commercially available software packages include Basama Perfusion 3.0.4.8 program (Kanazawa, Ishikawa, Japan); Perfusion CT software (Perfusion 2.0, GE Healthcare, Little Chalfont, Buckinghamshire, UK) with a body tumor perfusion algorithm; AZ-700W (Anzai Medical Co, Ltd, Tokyo, Japan), Syngo Body Perfusion CT (Syngo 2006G; Siemens Medical Solutions, Forchheim, Germany). For example, Perfusion CT software facilitates MDCT data with distributed parameter analysis; Siemens Medical Solutions implements MDCT data with Patlak analysis.

\section{Functional imaging using MDCT and color-encoded parameter display}

Functional imaging using MDCT (f-MDCT) has been proposed and evaluated for quantification of the perfusion and enhancement patterns of liver. ${ }^{37,38}$ By detecting the temporal changes in contrast enhancement from a series of images

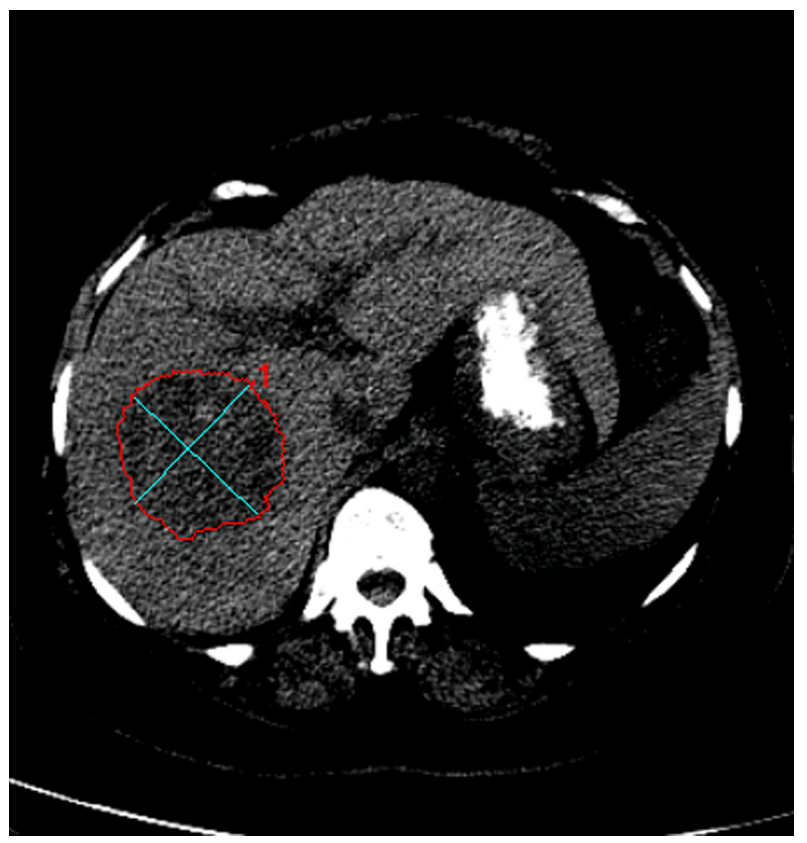

Figure I Automated delineation of colorectal cancer liver metastases achieved using a computer-assisted algorithm. ${ }^{33,173}$ On the axial precontrast CT image, the hepatic lesion segmented contours and perpendicular diameters $(6.54 \mathrm{~cm} \times 6.32 \mathrm{~cm})$ were obtained and overlapped. acquired over time, MDCT can trace tissue and vascular enhancement at small time intervals to capture homodynamic perfusion parameters that reflect useful information on (neo) angiogenesis within a liver lesion for qualitative and quantitative analysis. ${ }^{20,21}$ Semiquantitative assessments of vascular physiology may be obtained easily. ${ }^{39}$ The tracer kinetic modeling techniques, such as dual-input single-compartment model, and deconvolution techniques, such as the Johnson/ Wilson model, determine liver arterial and portal perfusion, leakage (extracellular) space, capillary permeability, and blood flow/volume. ${ }^{18,40-43}$

The definitions of semiquantitative/quantitative parameters were summarized in the literature. ${ }^{44}$ The definitions of the parameters are not further explained in this article. Semiquantitative parameters, such as CT attenuation $\left(H_{0}\right)$, time of arrival of contrast agent $\left(T_{0}\right)$, relative enhancement, area under the contrast concentration time curve (AUC), wash-in rate, wash-out rate, time between $T_{0}$ and time of peak CT attenuation (TTP), and brevity of enhancement, are easily derived from CT time-attenuation curve. Quantitative parameters, such as volume transfer constant $\left(K^{\text {trans }}\right)$, rate constant describing transfer of plasma to interstitium space $\left(K_{\mathrm{ep}}\right)$, extracellular extravascular space $\left(V_{\mathrm{e}}\right)$, vascular permeability-surface area product (PS), blood volume (BV), arterial blood flow $(\mathrm{ABF})$, arterial blood volume (ABV), portal blood flow (PBF), total blood flow (TBF), arterial perfusion rate (APR), hepatic perfusion index (HPI), extraction fraction (EF), and mean transit time (MTT), are obtained by using the compartment pharmacokinetic models such as dual-input single-compartment model and a deconvolution technique..$^{17,41,42,45,46}$ These parameters are very useful for the estimation of flow through the vessels, venous outflow vascular resistance, capillary wall permeability, composition of the extracellular space, and microvessel density. Standard models require a time course for the plasma concentration of iodine (usually referred to as the arterial input function and portal-venous input function) to calculate the quantitative parameters from CT time-attenuation curve. Due to direct linear relationship between CT attenuation value (expressed in Hounsfield units [HU]) and the plasma concentration of iodine, arterial/portal-venous input function can be measured from a conveniently placed artery/portal vein, respectively. $V_{\mathrm{e}}$ and relative enhancement are highly reproducible on f-MDCT. $K^{\text {trans }}, K_{\text {ep }}$, and wash-in/wash-out rate are sufficiently reproducible but lesser reproducible when compared with $V_{\mathrm{e}}$ and relative enhancement. ${ }^{20} \mathrm{~A}$ study suggested maximum slope CT perfusion is more suitable and reproducible than deconvolution CT perfusion. ${ }^{20}$ 
Morphological and functional MDCT can be a pixel-wise color-encoded display of various parameters derived from the quantitative data of the CT time-attenuation curve or the compartment pharmacokinetic model. This approach provides an automatic conversion of kinetic contrast information into easy-to-interpret color-scaled images, which provides a quick overview of the degree and heterogeneity of enhancement within the tissue. Pixel-displayed color-coded parameter imaging allows visualization of both anatomical morphology and the enhanced pharmacokinetic parameters, such as blood flow, permeability, leakage space in tumors, and blood volume. Color-coded display of various parameters provides unique insights into tumor structure, function and aggressiveness, and delivery of molecules into the space of Disse, and may therefore be helpful in guiding therapeutic intervention and biopsy sampling and monitoring treatment response. Colorcoded parameter images can provide detailed information of the enhancement pattern of focal and diffuse disease processes. Operator-defined region of interest (ROI) analysis can be developed by means of CT time-attenuation curves obtained by placing ROIs on color-coded parameter images. However, color-coded functional images have relatively poor signal-to-noise ratios. The poor signal-to-noise ratios can be overcome by fusing on morphological MDCT.

\section{Morphological and functional MDCT for hepatic disease and drug discovery}

The currently used nomenclature for hepatocellular focal liver lesions identifies 2 types of liver lesions: regenerative and neoplastic or dysplastic lesions. ${ }^{44,47-49}$ Regenerative lesions include regenerative nodules, segmental or lobar hyperplasia, and focal nodular hyperplasia (FNH). Neoplastic or dysplastic lesions include hepatocellular adenoma, dysplastic foci, dysplastic nodules, and hepatocellular carcinoma (HCC). Common hepatocellular lesions with benign growth behavior include cysts, hemangiomas, FNH, and hepatocellular adenomas, although the latter is rarely associated with malignant transformation. ${ }^{49}$ Diffuse liver parenchymal abnormalities include a wide variety of disease entities, such as hepatitis, cirrhosis, hemochromatosis, steatosis, and diffuse malignancies.

\section{Characterization and detection of focal liver lesions}

The vast majority of hemangiomas show a typical peripheral nodular enhancement pattern on relative-enhancement image from hepatic arterial phase and persistent fill-in of the entire lesion over time on relative-enhancement image from hepatic delayed phase. Characteristically, liver adenomas show a transient blush on the relative-enhancement image from hepatic arterial phase, and fade to iso-attenuation on the relative-enhancement image from hepatic delayed phase. In case of previous hemorrhage, adenoma can be inhomogeneous on the relative-enhancement image from hepatic arterial phase. Typically, FNH shows very intense homogeneous enhancement on the relative-enhancement image from hepatic arterial phase and iso-attenuation on the relative-enhancement image from hepatic delayed phase. The central scar of FNH is enhanced on the relative-enhancement image from hepatic delayed phase. Dysplastic nodules, especially high-grade dysplastic nodules, are premalignant lesions that can demonstrate enhancement on the relative-enhancement image from hepatic arterial phase. HCC (also called malignant hepatoma) typically shows intense and early enhancement on wash-in rate, $T_{0}$, TTP, and relative-enhancement image from hepatic arterial phase, and much of the contrast lost on wash-out rate or relative-enhancement image from hepatic delayed phases. In hepatic delayed phases, many HCCs show enhancement of a tumor capsule. ${ }^{23,44}$ Relatively higher and more variable perfusion parameters (BF, BV, HPI, AP, PS) and a lower MTT and PP values compared with background liver were observed in well-differentiated HCC compared with those in moderately/poorly differentiated (angioinvasive) HCC. ${ }^{37,50}$ Sahani et $\mathrm{al}^{17}$ demonstrated that $\mathrm{f}-\mathrm{MDCT}$ parameters $(\mathrm{BF}$, $\mathrm{BV}, \mathrm{PS}, \mathrm{MTT})$ are reproducible and have good correlation $(r=0.9)$ in quantifying tumor vascularity and angiogenesis in advanced HCC. Fibrolamellar HCC may show some overlap with FNH, including arterial enhancement and presence of a fibrous central scar. Important differences between FNH and fibrolamellar HCC include the following: (1) on relative-enhancement image from hepatic arterial phase, a more pronounced and homogeneous enhancement for $\mathrm{FNH}$, and a heterogeneous, less pronounced enhancement for fibrolamellar HCC; and (2) on relative-enhancement image from hepatic delayed phase, presence of washout in fibrolamellar HCC and persistent higher CT attenuation in FNH. The degree of enhancement of hypovascular liver metastases can be similar to the surrounding liver on the relative-enhancement image from hepatic arterial phase, but hypovascular liver metastases typically show lower enhancements than the surrounding liver and often show a peripheral irregular ring of enhancement on the relative-enhancement image from hepatic portal phases. The periphery of liver metastases often has a higher wash-in rate than the center of the lesion on imaging. Hypovascular liver metastases shows 
slightly delayed enhancement, and the liver lost some of its contrast. Therefore, the conspicuity of lesion has decreased. Hypervascular liver metastases are derived from highly vascular tumors, such as breast carcinoma, thyroid carcinoma, carcinoid, islet cell tumor, renal carcinoma, melanoma, pheochromocytoma. Small liver metastases often show intense enhancement, whereas, large liver metastases may only display enhancement in the peripheral rim. Significant changes in perfusion parameters (earlier $T_{0} / \mathrm{TTP}$, increased $\mathrm{ABF} / \mathrm{ABV} / \mathrm{HPI} / \mathrm{PS} /$ wash-in rate) were color-coded image in hypervascular liver metastases owing to the increased hepatic arterial component, as well as arteriovenous shunting in tumor. ${ }^{20,51-54}$ Metastases (micro) can be identified as areas of high perfusion on HPI image..$^{53,55,56} \mathrm{f}$-MDCT even display occult hepatic metastases. ${ }^{40}$ The increase in ABF within the metastasis (especially the adjacent liver) is correlated with the patient's survival time. ${ }^{52}$

Reported sensitivity of MDCT varies widely, with values of $6 \%-89 \%$ for the detection of HCC. ${ }^{57,58}$ Relatively high sensitivity $(74 \%-85 \%)$ for metastatic liver tumors has been reported. ${ }^{58}$ Primary and metastatic liver malignancies and cirrhosis can be earlier detected based on relative increases in HBF. Diagnostic sensitivity and accuracy of MDCT are significantly improved and result in better detection of hepatic lesions, thus decreasing the number of biopsies. ${ }^{23}$

\section{Evaluation of diffuse liver disease and hepatic perfusion disorder}

Diffuse liver parenchymal diseases consist of various disease processes, such as cirrhosis, infectious and inflammatory diseases, storage diseases, vascular diseases, and diffuse malignancies. ${ }^{59}$ Significant changes in perfusion parameters (increased $\mathrm{ABF} / \mathrm{BV} / \mathrm{MTT}$, and decreased $\mathrm{PBF} / \mathrm{TBF}$ ) were observed in cirrhosis as a result of excessive deposition of collagen in the space of Disse and defenestration of the basal lamina, sinusoids. 22,23,60-62 Moreover, the changes in the perfusion parameters correlated with the severity (the degree of fibrosis) of chronic liver disease. Acute hepatitis may present with heterogeneous patchy enhancement of the affected liver parenchyma in hepatic arterial phase and becomes occult in hepatic portal and delayed phases. MDCT have been applied to evaluate hepatic functional reserve and liver volume variation in patients with chronic liver diseases. ${ }^{63-64}$ Hepatic perfusion disorders are related to a variety of disease entities or anatomic variants, such as portal venous obstruction, arterial obstruction, hepatic venous obstruction (eg, Budd-Chiari syndrome, heart failure, mediastinal fibrosis), mediastinal or thoracic venous inlet obstruction, focal liver lesions, inflammatory processes, normal anatomic variants in the hepatic blood supply, altered hemodynamics after the placement of a transjugular intrahepatic portosystemic shunt, and uncertain causes. ${ }^{53,65}$ Hepatic perfusion disorders (except siphoning effect, severe hepatic artery stenosis or occlusion, hepatic infarct, and Budd-Chiari syndrome) typically presents high enhancement of the affected liver parenchyma on the relative-enhancement image from hepatic arterial phase, and iso-enhancement of the affected liver parenchyma on the relative-enhancement image from hepatic portal phase. Arterioportal shunt shows early enhancement of the peripheral portal vein branches and transient enhancement of peripheral wedge-shaped affected liver parenchyma with a clear margin on the relative-enhancement image from hepatic arterial phase. Severe hepatic artery stenosis or occlusion and hepatic infarction typically demonstrate a wedge-shaped diminished enhancement of the affected liver parenchyma on the relative-enhancement image from hepatic arterial phase and hepatic portal phase, respectively. Budd-Chiari syndrome demonstrate heterogeneous and mosaic enhancement pattern and absence of hepatic vein enhancement on the relative-enhancement image from hepatic arterial phase and from hepatic portal phase.

\section{Tumor staging and prognosis}

The tumor-node-metastasis (TNM) staging system is widely used to stage liver cancer. The up-to-date edition of AJCC Cancer Staging Manual becomes effective in 2010 (Table 1). ${ }^{66}$ Morphological and functional MDCT findings compose crucial elements in defining the TNM stage of HCC. MDCT provided the accurate stage of disease in $46 \%$ of cirrhotic candidates for liver transplantation. ${ }^{57}$ Progressive disease had lower baseline MTT values and a higher percent increase after bevacizumab therapy than stable disease or partial response in patients with advanced HCC. ${ }^{67}$

\section{Monitoring therapy response and novel drug discovery programs}

Accurate therapy response assessment is important for judging the outcome of therapy. ${ }^{68-70}$ Early selection of patients who are most likely to benefit from a specific type of therapy would prevent time consumption, money, and unnecessary drug toxicity in nonresponding patients. ${ }^{1}$ Direct pathological measurement of tumor, such as tumor microvessel density (MVD), in biopsy specimens provides potentially unreliable information of the vessel function. ${ }^{71}$ Together with other relevant biomarkers and genomics, morphological and functional MDCT, based on therapy response criteria, has been 
Table I Tumor node metastasis staging of liver cancer (American Joint Committee on Cancer 7th edition, 2010)

\section{Primary tumor (T)}

TX: Primary tumor cannot be assessed

T0: No evidence of primary tumor

TI: Solitary tumor without vascular invasion

T2: Solitary tumor with vascular invasion or multiple tumors none more than $5 \mathrm{~cm}$

T3a: Multiple tumors more than $5 \mathrm{~cm}$

T3b: Single tumor or multiple tumors of any size involving a major branch of the portal or hepatic veins

T4: Tumor(s) with direct invasion of adjacent organs other than gallbladder or with perforation of visceral peritoneum

Regional lymph nodes (N)

NX: Regional lymph nodes cannot be assessed

No: No regional lymph node metastasis

NI: Regional lymph node metastasis

\section{Distant metastasis (M)}

MX: Distant metastasis cannot be assessed

M0: No distant metastasis

MI: Distant metastasis

Note: The system only applies to hepatocellular carcinoma staging and no longer applies to intrahepatic cholangiocarcinoma staging. Sarcoma and tumors metastatic to the liver are not included. Inferior phrenic lymph nodes were reclassified into regional lymph nodes from distant lymph nodes. Copyright $\odot$ 2010. Reprinted with permission from Edge SB, Byrd DR, Carducci MA, Compton CC, editors. AJCC Cancer Staging Handbook. 7th ed. New York: Springer-Verlag; 2010.

Abbreviation: TNM, tumor node metastasis.

applied to assess early response to novel targeted therapies and antitumor activity in novel drug discovery programs.

Two major systems of response measurement and classification are widely used in the morphologic assessment of tumor response: the World Health Organization (WHO) criteria and the Response Evaluation Criteria in Solid Tumors (RECIST) (Table 2). ${ }^{72-76}$ The up-to-date RECIST (version 1.1) becomes effective in $2009 .{ }^{75}$ These systems rely solely on the changes in tumor size on serial examinations performed before, during, and after chemotherapy (Table 2). Such information is essential to determine whether the therapy is beneficial to the patient, to help make clinical decisions, and for the development and approval of new anticancer drugs. However, the WHO/RECIST criteria have not taken functional or volumetric information into consideration. Firstly, in tumor size due to tumor shrinkage in one or two dimensions may not always provide adequate and timely information on tumor response to therapy. Morphologic findings may lag chronologically or even fail to correspond to the patient's clinical response and functional changes in tumors, and thus may not be able to adequately capture the effects of novel therapies. Secondly, in tumor size may not provide accurate prognosis for an individual patient. RECIST alone cannot be used to evaluate local treatment of tumors by cryoablation or radiofrequency ablation. ${ }^{77-81}$ Moreover, recently, the new agents such as cytostatic (halting tumor growth) agents, epidermal growth factor receptor, tyrosine kinase inhibitors, such as gefitinib and erlotinib, or antiangiogenic (inhibiting or disrupting tumor neovasculature growth) agents, such as bevacizumab (Avastin, Genentech/ Roche), have been shown to increase survival in patients with hepatic malignancies when combined with standard cytotoxic (causing tumor shrinkage) therapies. ${ }^{82}$ After being treated with these newer agents, tumors respond by undergoing cystic change and central necrosis that may not be captured by the WHO or RECIST response criteria. ${ }^{60,83}$ Modified response criteria for patients with HCC and gastrointestinal stromal tumor incorporate tumor functional information and the extent of macroscopic tumor necrosis or cystic changes on imaging such as MDCT that could improve conventional RECIST criteria ${ }^{84}$ European Association for the Study of the Liver (EASL) criteria uses the reduction in viable tumor volume to assess tumor response by using the reduction in viable tumor volume.$^{85}$ Residual tumor and the extent of tumor necrosis can be evaluated using EASL.

Tumor morphological change on MDCT is important information to monitor therapy response and novel drug discovery programs. A study of 68 patients with metastatic colorectal cancer on clinical trials of hepatic arterial infusion of floxuridine and dexamethasone with systemic oxaliplatin with $5 \mathrm{FU} / \mathrm{LV}$ and/or irinotecan demonstrated that there was a $92.6 \%$ agreement rate and a $7.4 \%$ disagreement rate in best overall response between RECIST and WHO. Mean percentage decrease in tumor size among the 27 unidimensional responders and 28 bidimensional responders was $65.0 \%$ and $79.7 \%$, respectively. Mean increase in the tumor size among the 36 unidimensional progressors and 48 bidimensional progressors was $61.8 \%$ and $139.1 \%$, respectively. The average robustness value was $-35.3 \%$ by RECIST and $53.9 \%$ by WHO among the 17 discordant pairs and $-25.9 \%$ by RECIST and $78.9 \%$ by WHO among the 51 concordant pairs. A comparison of robustness of response in time to best overall response found an average robustness value of $35.7 \%$ by RECIST vs $24.8 \%$ by WHO among the 10 discordant cases and $5.3 \%$ by RECIST vs $60.6 \%$ by WHO among the 58 concordant cases. Time to tumor progression was different between WHO and RECIST $(P=0.006)$. The authors concluded that this may impact clinical trials, whose results are interpreted with RECIST and WHO. A new method of measuring robustness of response may indicate disease sites or therapies that are affected by measurement technique, and help to define the degree of response or progression. ${ }^{35} \mathrm{~A}$ study of 70 patients demonstrated that the optimal duration of chemotherapy (significant reduction 
Table 2 Comparison of WHO response criteria and RECIST I. 0 and RECIST I. I2,74,75,174 $^{\text {Re }}$

\begin{tabular}{|c|c|c|c|}
\hline & WHO & RECIST I.0 & RECIST I.I \\
\hline \multirow[t]{3}{*}{$\begin{array}{l}\text { Tumor size } \\
\text { calculation } \\
\text { methods }\end{array}$} & $\begin{array}{l}\text { Measurable: } \\
\text { Bidimensional (the cross product } \\
\text { of the longest diameter and the } \\
\text { longest perpendicular diameter). } \\
\text { Lesion number: not specified. }\end{array}$ & $\begin{array}{l}\text { Measurable: } \\
\text { Unidimensional } \\
\text { (the longest diameter) } \\
\text { Lesion size: } \geq 10 \mathrm{~mm} \\
\text { (spiral CT); } \geq 20 \mathrm{~mm} \\
\text { (nonspiral CT); } \\
\text { Lesion number: } \leq 10 \text { lesions } \\
\text { (5/organ). }\end{array}$ & $\begin{array}{l}\text { Measurable: } \\
\text { Unidimensional ( the longest diameter) } \\
\text { Lesion Size: } \geq 10 \mathrm{~mm} \text { (spiral CT slice thickness } \\
\leq 5 \mathrm{~mm} \text { ); or } 2 \times \text { slice thickness (slice thickness } \\
>5 \mathrm{~mm} \text { ); } \geq 20 \mathrm{~mm} \text { (nonspiral CT). } \\
\text { Lymph node: } \geq 15 \mathrm{~mm} \text { short axis for target, } \\
\geq 10<15 \mathrm{~mm} \text { for non-target, } \\
<10 \mathrm{~mm} \text { is non-pathological Lesion Number: } \geq 5 \\
\text { lesions ( } 2 \text { organ). }\end{array}$ \\
\hline & $\begin{array}{l}\text { Nonmeasurable: } \\
\text { e.g, lymphangitic pulmonary metastases. }\end{array}$ & $\begin{array}{l}\text { Nonmeasurable: } \\
\text { All other lesions including small } \\
\text { lesions. }\end{array}$ & $\begin{array}{l}\text { Nonmeasurable: } \\
\text { All other lesions including } \\
\text { small lesions. }\end{array}$ \\
\hline & $\begin{array}{l}\text { Measurable: } \\
\text { PD: } \geq 25 \% \text { increase of at least one } \\
\text { measurable lesion or } \\
\text { appearance of new lesions; CR: } \\
\text { disappearance of all known disease } \\
\text { (confirmed at least } 4 \text { wk later); } \\
\text { PR: } \geq 50 \% \text { decrease from baseline } \\
\text { (confirmed at least } 4 \text { wk later); } \\
\text { NC: neither PR nor PD criteria met. }\end{array}$ & $\begin{array}{l}\text { Target: } \\
\text { PD: } \geq 20 \% \text { increase in sum of longest } \\
\text { diameter from nadir or } \\
\text { appearance of new lesions; CR: } \\
\text { disappearance of all known disease } \\
\text { (confirmed at least } 4 \text { wk later); } \\
\text { PR: } \geq 30 \% \text { decrease from baseline } \\
\text { (confirmed at least } 4 \text { wk later); } \\
\text { SD: neither PR nor PD criteria met. }\end{array}$ & $\begin{array}{l}\text { Target: } \\
\text { PD: } \geq 20 \% \text { increase in sum of } \\
\text { longest diameter from nadir and } \geq 5 \mathrm{~mm} \\
\text { net increase or appearance of new lesions; } \\
\text { CR: disappearance of all known disease } \\
\text { (confirmed at least } 4 \text { wk later); PR: } \geq 30 \% \\
\text { decrease from baseline (confirmed at least } \\
4 \text { wk later); SD: neither PR nor PD criteria met } \\
\text { Lymph node: }<10 \text { mm short axis is CR }\end{array}$ \\
\hline $\begin{array}{l}\text { Response } \\
\text { criteria }\end{array}$ & $\begin{array}{l}\text { Nonmeasurable: } \\
\text { PD: } \geq 25 \% \text { increase } \\
\text { of existent lesions or new lesions; } \\
\text { CR: disappearance of all known } \\
\text { disease (confirmed at least } 4 \text { wk later); } \\
\text { PR: } \geq 50 \% \text { decrease from baseline } \\
\text { (confirmed at least } 4 \text { wk later); NC: } \\
\text { neither PR nor PD criteria met. }\end{array}$ & $\begin{array}{l}\text { Nontarget: } \\
\text { PD: unequivocal progression or } \\
\text { appearance of new lesions; CR: } \\
\text { disappearance of all nontarget } \\
\text { lesions and normal tumor markers } \\
\text { (confirmed at least } 4 \text { wk later); } \\
\text { non-PD: persistence of one or more } \\
\text { nontarget lesions or tumor marker } \\
\text { abnormal elevation. }\end{array}$ & $\begin{array}{l}\text { Nontarget: } \\
\text { PD: unequivocal progression } \\
\text { (eg, } 75 \% \text { increase in volume); or new } \\
\text { "positive PET" scan with confirmed anatomic } \\
\text { progression) or appearance of new lesions; } \\
\text { CR: disappearance of all nontarget lesions } \\
\text { and normal tumor markers (confirmed at } \\
\text { least } 4 \text { wk later); non-PD: persistence of one } \\
\text { or more nontarget lesions or tumor marker } \\
\text { abnormal elevation. }\end{array}$ \\
\hline
\end{tabular}

Abbreviations: WHO, World Health Organization; RECIST, Response Evaluation Criteria in Solid Tumors; CT, computed tomography; PD, progressive disease; $\mathrm{CR}$, complete response; PR, partial response; NC, no change.

in tumor size on MDCT scans) was 2-4 months prior to liver resection for colorectal liver metastases. ${ }^{86}$ In a phase 2 study of sorafenib (an oral multikinase inhibitor that targets Raf kinase and receptor tyrosine kinases) in 137 patients with inoperable HCC, baseline and serial follow-up MDCT scans demonstrated tumor necrosis, overall tumor response, time to progression, and overall survival. ${ }^{87,88} \mathrm{~A}$ study of 23 patients with advanced HCC treated with MDCT and sunitinib after radiation therapy demonstrated a high RECIST with an objective response in $74 \%$ of patients. ${ }^{89}$ A study of 34 patients with metastatic colorectal cancer on clinical trials of floxuridine and dexamethasone with 5FU/ LV or intravenous oxaliplatin and irinotecan demonstrated a 97\% agreement rate in best overall response between unidimensional and bidimensional measurements. Time to progression was 9.3 months measured unidimensionally compared with 7.6 months measured bidimensionally. Time to nadir or best overall response was slightly longer in unidimensional measurements compared with the bidimen- sional measurements. The authors concluded that there is a high concordance in the best overall response classification between WHO and RECIST. However, time to progression and time to best overall response/nadir is different. This information should be considered in clinical trial design, clinical trial interpretation, in the interpretation of a given patient's response (or apparent lack of response) to therapy, and in the development of anticancer drugs. ${ }^{90}$ This work provided a new avenue for study of colorectal carcinoma therapy assessment, which had troubled colorectal carcinoma research and clinical practice for decades and had a great impact on the research and clinical practice in this field worldwide.

Tumor functional change, such as the decrease in hemodynamic blood flow parameters and tumor microvessel density on f-MDCT, after administration of anti-angiogenesis drugs (bevacizumab, cediranib [Recentin ${ }^{\circledR}$, AZD2171, AstraZeneca plc, Westminster, London], SU6668 [Pfizer, New York, USA], Sorafenib [Nexavar ${ }^{\circledR}$; Bayer Healthcare], 
and sunitinib [Sutent, SU11248]), antivascular endothelial growth factor (receptor) or platelet-derived growth factor receptor (VEGF(R)/PDGFR) agents, or antivascular drugs (such as endostatin) is concordant with that on other imaging studies, such as magnetic resonance imaging (MRI) or positron emission tomography (PET). ${ }^{20,91,92}$ Functional changes may be earlier or more specific than anatomic morphological changes after antiangiogenic/vascular or anti-VEGF(R)/PDGFR drugs. ${ }^{18}$ The use of f-MDCT provides accurate quantification of perfusion function of mature vessels without changes of MVD after antiangiogenic treatment with SU5416. ${ }^{93}$ In a study of 16 patients with liver metastatic lesion of renal cell carcinoma after thalidomide therapy, the decrease in perfusion parameters (BF, BV, MTT, PS) were color-coded imaged, time to progression correlated with $\mathrm{BF}$ and $\mathrm{PS}$, and responders had a higher decrease in BF than the nonresponders. ${ }^{54} \mathrm{~A}$ study of 33 patients with measurable locally advanced, recurrent, or metastatic $\mathrm{HCC}$ demonstrated a significant decrease in BF, $\mathrm{BV}$, and PS in HCC and an increase in MTT on f-MDCT after bevacizumab therapy ${ }^{67}$ Quantification of hepatic blood flow on f-MDCT may also be useful to assess vascular changes secondary to interventional therapies, earlier identification of tumor recurrence, and liver graft rejection in transplant patients. ${ }^{23,94}$ The substantial increase in ABF and TBF (but PBF remained unchanged) in transjugular intrahepatic portosystemic shunt placement was observed on $\mathrm{f}-\mathrm{MDCT} .{ }^{60} \mathrm{f}$-MDCT provides accurate and reproducible quantification of liver tumor perfusion after transcatheter arterial chemoembolization procedure. ${ }^{95} \mathrm{~A}$ study of 13 patients treated with a combination of AZD2171 (AstraZeneca, Wilmington, Delaware, USA) and gefitinib (Iressa ${ }^{\circledR}$, ZD1839; AstraZeneca) demonstrated an initial decrease of $39 \%$ in wash-in rate within the liver tumors and $36 \%$ in wash-in rate within a rim region surrounding the tumors, followed by a trend toward recovery of hepatic artery flow detected by f-MDCT. ${ }^{20}$

\section{Partial liver resection and transplantation}

Morphological and functional MDCT have been applied to evaluate hepatic function in partial liver resection and transplantation. ${ }^{96,97} \mathrm{CT}$ volumetry, in which liver volume is the established method to assess future remnant liver function assessment before partial hepatectomy, follow-up after preoperative portal vein embolization, postoperative liver regeneration, and graft function. ${ }^{98-105}$ However, the correlation coefficient between the postoperative indocyanine green (ICG) clearance predicted by MDCT and the actual postoperative ICG clearance is moderate. ${ }^{106,107}$ Three-dimensional algorithms have been developed to calculate metabolic liver volume and nonmetabolic liver volume on MDCT. ${ }^{102,108,109}$

\section{MDCT-guided interventions}

MDCT-guided interventional methods have been applied to deliver personalized therapies locally in the human body. ${ }^{1,110-113}$

\section{Parallel imaging modalities and "fused" imaging modalities}

As with MDCT, several imaging modalities, such as ultrasound, MRI, single-photon emission computed tomography (SPECT), and PET, have been used for hepatic disease and drug discovery. Ultrasound can be applied for quantification by means of Doppler ultrasonography or contrast-enhanced ultrasound or ultrasound elasticity. ${ }^{114,115}$ However, the main limitation of ultrasound examinations is that it is operator dependent, hence with low reproducibility and has low sensitivity. ${ }^{116}$ Doppler ultrasound assesses morphological and hemodynamic changes of liver circulation. With the introduction of second-generation microbubble contrast media, contrast-enhanced ultrasound characterizes enhancement patterns of hepatic lesions, similar to that achieved with f-MDCT and dynamic contrast-enhanced MRI (DCE-MRI). Transient elastography (FibroScan ${ }^{\mathrm{TM}}$, Echosens, Paris, France) is a novel method for the evaluation of hepatic fibrosis in patients with chronic liver diseases. ${ }^{117-119}$ MRI has superior soft-tissue contrast resolution, lacks ionizing radiation, and has the possibility of performing functional and metabolic imaging sequences, which are particularly advantageous for serial examinations to monitor therapy response and the whole liver surveillance of HCC and hepatic dysplastic nodules. Compared with MRI, scintigraphy, and PET, MDCT has the highest spatial resolution, it is less cost and time consuming, and it is broadly available. However, radiation doses must be considered. The choice between MDCT and MRI depends on machine availability, doctor expertise, tumor location, desired parameters, and the requirement of decreased radiation burden. ${ }^{120,121}$ DCE-MRI, with f-MDCT, studies hepatic perfusion, tumor capillary wall permeability, and biliary excretion using different kinds of contrast media. ${ }^{44,122-124}$ Both DEC-MRI and f-MDCT are easily incorporated into routine examinations. ${ }^{120}$ There are differences between the 2 imaging modalities in acquisition techniques, mathematical analysis, and propensity to artifacts. ${ }^{120} \mathrm{f}-\mathrm{MDCT}$ may be more 
quantitative and more reproducible than DCE-MRI. ${ }^{120,125}$ Diffusion-weighted imaging (DWI) and diffusion tensor imaging measure the Brownian motion of water molecules in biologic tissues. ${ }^{126,127}$ The apparent diffusion coefficient (ADC) derived from DWI and diffusion tensor imaging, has been in use for the quantitative assessment of tissue diffusivity. DWI demonstrated no or minimal signal decrease from $b$ of $0 \mathrm{~s} / \mathrm{mm}^{2}$ to a higher $b$ value, reduced $\mathrm{ADC}$, and increased fractional anisotropy in malignant lesion. ${ }^{128-134} \mathrm{DWI}$ and diffusion tensor imaging still have poor SNR, limited spatial resolution, and ADC reproducibility. ${ }^{135,136}$ MR spectroscopy (MRS) investigates metabolism for diffuse and chronic liver diseases, congenital diseases, diabetes, and cancer. ${ }^{137-139}$ MRS can quantify liver fat by measuring lipid peaks and identify malignancy, by measuring choline peak, lactate/pyruvate ratios, and diagnose liver dysfunction by measuring $31 \mathrm{P}$ metabolites. ${ }^{140-145}$ MRS imaging has a rather low spatial resolution and still mainly used as a clinical research tool. MR elastography noninvasively assesses the viscoelasticity of tissues and has been applied to detect and stage liver fibrosis, which increases hepatic stiffness or elasticity. ${ }^{146-149}$ SPECT is obtained after intravenous injection of a radiopharmaceutical agent, such as technetium 99m-labeled, sulfur, pertechnetate, DTPAgalactosyl albumin. ${ }^{150-158}$ 99m Tc-GSA scintigraphy (receptor mediated) and ${ }^{99 \mathrm{~m}} \mathrm{Tc}-$-mebrofenin HBS (hepatic uptake and excretion) reliably assess hepatic function and functional reserve. ${ }^{150-156}$ Several studies demonstrated that ${ }^{99 \mathrm{~m}} \mathrm{Tc}-\mathrm{GSA}$ scintigraphy has a strong correlation with ICG clearance test and holds incremental value over $\mathrm{CT}$ volumetry for predicting remnant liver function. ${ }^{106,150}$ Metabolic imaging with PET is performed after the intravenous injection of radiotracers, such as ${ }^{18} \mathrm{FDG}, 11 \mathrm{C}$-acetate, $62 \mathrm{Cu}-\mathrm{PTSM}$, and $\mathrm{C} 15 \mathrm{O}$ 2, to evaluate cancer metabolism, endocrine status, hypoxia, and oncofetal and differentiation antigens. ${ }^{159-162}$ The degree of changes in tumor glucose metabolism determined by $18 \mathrm{~F}-\mathrm{FDG}$ PET is predictive of patient outcome. Semiquantitative measurements (eg, standardized uptake value [SUV]) and full kinetic quantitative analyses have been developed to measure the rate of glucose metabolism. ${ }^{159}$ SPECT and PET have inadequate spatial resolution compared with MRI and CT.

"Fused" or "hybrid" imaging modalities, such as ultrasonography/CT, SPECT/CT, PET/CT, and f-CT/PET/CT, integrate advantages of different modalities yielding comprehensive information regarding changes in liver disease at different levels (anatomic, metabolic, molecular, histologic, and other levels). ${ }^{163-165}$ PET/MRI is the next generation of clinical imaging multimodality, which may not only provide the potential to provide more anatomical, functional, metabolic, and molecular details than MDCT, especially when scanning soft tissue, but also considerably lower radiation exposure compared with PET/CT. ${ }^{166-171}$ However, the development of PET/MRI system is technically more challenging, and the cost of PET/MRI would be likely higher. ${ }^{166,172}$

In summary, as the era of personalized medicine is approaching, morphological and functional MDCT, together with other relevant biomarkers and genomics, serves as a problem-solving tool and surrogate biomarker for hepatic disease characterization, detection, tumor staging and prognosis, therapy response assessment, novel drug discovery programs, partial liver resection and transplantation, and CT-guided interventions. Analysis of color-coded images offers an easy-to-interpret presentation of hepatic disease behavior changes on blood flow, permeability, leakage space, and blood volume. Fused imaging modalities, such as ultrasonography/CT, SPECT/CT, PET/CT, and f-CT/PET/CT, yield comprehensive information regarding changes in liver disease at different levels (anatomic, metabolic, molecular, histologic, and other levels). It is critical for the development of computational image analysis techniques for MDCT to detect and define liver cancer, which would be helpful in presurgical planning for hepatic resection and to gauge the results of therapies.

\section{Conclusion}

In the era of personalized medicine, advanced morphological and functional MDCT in combination with imaging postprocessing algorithms, together with other relevant biomarkers and genomics, serves as a noninvasive problem-solving tool and surrogate biomarker to effectively improve hepatic disease characterization, detection, tumor staging prognosis, therapy response assessment, novel drug discovery programs, partial liver resection and transplantation, and CT-guided interventions.

\section{Abbreviations}

$T_{0}$, time of arrival of contrast agent; $H_{0}$, CT attenuation (expressed in HU) prior to contrast agent; TTP, time between $T_{0}$ and time of peak CT attenuation; AUC, area under the contrast concentration time curve; IAUC, initial area under the contrast concentration time curve; $\mathrm{BF}$, blood flow; $\mathrm{ABF}$, arterial blood flow; PBF, portal blood flow; TBF, total blood flow; ABV, arterial blood volume; APR, arterial perfusion rate; HPI, hepatic perfusion index; AP, arterial perfusion; PP, portal perfusion; SPV, standardized perfusion values; BV, blood volume; MTT, mean transit time; PS, vascular permeability-surface area product; $V_{\mathrm{e}}$, extracellular extravascular space; $k_{\text {ep }}$ (or $\mathrm{K}_{21}$ ), rate constant describing 
transfer of plasma to interstitium space; rBV, relative blood volume; $k^{\text {trans }}$, volume transfer constant; $\mathrm{k}_{\mathrm{el}}$, rate of clearance of contrast agent from the vascular compartment; $K_{\mathrm{i}}$, bidirectional transfer constant; EF, extraction fraction; RECIST, Response Evaluation Criteria in Solid Tumors; WHO, World Health Organization; EASL, European Association for the Study of the Liver; VEGF(R), vascular endothelial growth factor (receptor); PDGFR, platelet-derived growth factor receptor; AZD2171, an oral tyrosine kinase inhibitor of the vascular endothelial growth factor receptor-2 (VEGFR-2) with additional inhibitory activity to VEGFR-1 and VEGFR3 ; thalidomide, inhibitor of TNF- $\alpha$, interleukins, and interferons; TNF, tumor necrosis factor; ROI, region of interest; AJCC, American Joint Committee on Cancer, f-MDCT, functional multidetector computed tomography; GIST, gastrointestinal stromal tumor; MVD, blood vessel density; TNM, tumor node metastasis; ICG, indocyanine green.

\section{Disclosure}

The author reports no conflict of interest in this work.

\section{References}

1. Thrall JH. Personalized medicine. Radiology. 2004;231(3):613-616.

2. Fackler JL, McGuire AL. Paving the way to personalized genomic medicine: steps to successful implementation. Curr Pharmacogenomics Person Med. 2009;7(2):125-132.

3. Kumar A, Ajilore O. Magnetic resonance imaging and late-life depression: potential biomarkers in the era of personalized medicine. Am J Psychiatry. 2008;165(2):166-168.

4. Smith JJ, Sorensen AG, Thrall JH. Biomarkers in imaging: realizing radiology's future. Radiology. 2003;227(3):633-638.

5. Hariri AR, Weinberger DR. Imaging genomics. Br Med Bull. 2003;65: 259-270.

6. Kapse N, Goh V. Functional imaging of colorectal cancer: positron emission tomography, magnetic resonance imaging, and computed tomography. Clin Colorectal Cancer. 2009;8(2):77-87.

7. Purdie TG, Henderson E, Lee TY. Functional CT imaging of angiogenesis in rabbit VX2 soft-tissue tumour. Phys Med Biol. 2001;46(12): 3161-3175.

8. Cao Y, Pan C, Balter JM, et al. Liver function after irradiation based on computed tomographic portal vein perfusion imaging. Int J Radiat Oncol Biol Phys. 2008;70(1):154-160.

9. Sahani DV, Kalva SP. Imaging the liver. Oncologist. 2004;9(4):385-397.

10. Oliver JH 3rd, Baron RL, Federle MP, Jones BC, Sheng R. Hypervascular liver metastases: do unenhanced and hepatic arterial phase CT images affect tumor detection? Radiology. 1997;205(3):709-715.

11. Silverman PM, Szklaruk J. Controversies in imaging of hepatocellular carcinoma: multidetector CT (MDCT). Cancer Imaging. 2005;5: 178-187.

12. Winterer JT, Kotter E, Ghanem N, Langer M. Detection and characterization of benign focal liver lesions with multislice CT. Eur Radiol. 2006;16(11):2427-2443.

13. Mori S, Obata T, Kishimoto R, et al. Clinical potentials for dynamic contrast-enhanced hepatic volumetric cine imaging with the prototype 256-MDCT scanner. AJR Am J Roentgenol. 2005; 185(1):253-256.

14. Johnson TR, Krauss B, Sedlmair M, et al. Material differentiation by dual energy CT: initial experience. Eur Radiol. 2007;17(6):1510-1517.
15. Kobayashi T, Hayashi T, Funabasama S, et al. Three-dimensional perfusion imaging of hepatocellular carcinoma using 256-slice multidetectorrow computed tomography. Radiat Med. 2008;26(9):557-561.

16. Ho LM, Yoshizumi TT, Hurwitz LM, et al. Dual energy versus single energy MDCT: measurement of radiation dose using adult abdominal imaging protocols. Acad Radiol. 2009;16(11):1400-1407.

17. Sahani DV, Holalkere NS, Mueller PR, Zhu AX. Advanced hepatocellular carcinoma: CT perfusion of liver and tumor tissue - initial experience. Radiology. 2007;243(3):736-743.

18. Dawson P. Functional imaging in CT. Eur J Radiol. 2006;60(3):331-340.

19. Laghi A. Multidetector CT (64 slices) of the liver: examination techniques. Eur Radiol. 2007;17(3):675-683.

20. Meijerink MR, van Cruijsen H, Hoekman K, et al. The use of perfusion CT for the evaluation of therapy combining AZD2171 with gefitinib in cancer patients. Eur Radiol. 2007;17(7):1700-1713.

21. Wang L, Hussain SM, Heerebeek BV, et al. Postprocessing of dynamic gadolinium-enhanced MRI exams of the upper abdominal organs: explanation and potential clinical application of color-coded qualitative and quantitative analysis. Radiology. 2000;217(P):635.

22. Nakashige A, Horiguchi J, Tamura A, et al. Quantitative measurement of hepatic portal perfusion by multidetector row $\mathrm{CT}$ with compensation for respiratory misregistration. Br J Radiol. 2004;77(921):728-734.

23. Pandharipande PV, Krinsky GA, Rusinek H, Lee VS. Perfusion imaging of the liver: current challenges and future goals. Radiology. 2005; 234(3):661-673.

24. Thomsen HS, Morcos SK. Contrast-medium-induced nephropathy: is there a new consensus? A review of published guidelines. Eur Radiol. 2006;16(8):1835-1840.

25. Regino CA, Walbridge S, Bernardo M, et al. A dual CT-MR dendrimer contrast agent as a surrogate marker for convection-enhanced delivery of intracerebral macromolecular therapeutic agents. Contrast Media Mol Imaging. 2008;3(1):2-8.

26. Kattumuri V, Katti K, Bhaskaran S, et al. Gum arabic as a phytochemical construct for the stabilization of gold nanoparticles: in vivo pharmacokinetics and X-ray-contrast-imaging studies. Small. 2007;3(2):333-341.

27. Simon GH, FuY, Berejnoi K, et al. Initial computed tomography imaging experience using a new macromolecular iodinated contrast medium in experimental breast cancer. Invest Radiol. 2005;40(9):614-620.

28. Fournier LS, Cuenod CA, de Bazelaire C, et al. Early modifications of hepatic perfusion measured by functional $\mathrm{CT}$ in a rat model of hepatocellular carcinoma using a blood pool contrast agent. Eur Radiol. 2004;14(11):2125-2433.

29. Masutani Y, Uozumi K, Akahane M, Ohtomo K. Liver CT image processing: a short introduction of the technical elements. Eur J Radiol. 2006;58(2):246-251.

30. Liu F, Zhao B, Kijewski PK, Wang L, Schwartz LH. Liver segmentation for CT images using GVF snake. Med Phys. 2005;32(12):3699-3706.

31. Zhao B, Schwartz LH, Jiang L, et al. Automatic measurements of hepatic metastases on contrast enhanced CT scan - comparison with radiologists' manual results. Radiology. 2004;233(P):277.

32. Keil S, Plumhans C, Behrendt FF, et al. Semi-automated quantification of hepatic lesions in a phantom. Invest Radiol. 2009;44(2):82-88.

33. Zhao B, Schwartz LH, Jiang L, et al. Shape-constraint region growing for delineation of hepatic metastases on contrast-enhanced computed tomograph scans. Invest Radiol. 2006;41(10):753-762.

34. Keil S, Behrendt FF, Stanzel S, et al. Semi-automated measurement of hyperdense, hypodense and heterogeneous hepatic metastasis on standard MDCT slices. Comparison of semi-automated and manual measurement of RECIST and WHO criteria. Eur Radiol. 2008;18(11): 2456-2465.

35. Wang L, Schwartz LH, Mazumdar M, Smith A, Kemeny.NE. robustness of response: a new measure of response assessment - its impact on metricies of response in colorectal cancer. J Clin Oncol. 2005; 3(16):263.

36. Mantatzis M, Kakolyris S, Amarantidis K, Karayiannakis A, Prassopoulos $\mathrm{P}$. Treatment response classification of liver metastatic disease evaluated on imaging. Are RECIST unidimensional measurements accurate? Eur Radiol. 2009;19(7):1809-1816. 
37. Miles KA, Hayball MP, Dixon AK. Functional images of hepatic perfusion obtained with dynamic CT. Radiology. 1993;188(2):405-411.

38. Lee TY, Purdie TG, Stewart E. CT imaging of angiogenesis. Q J Nucl Med. 2003;47(3):171-187.

39. Miles KA. Perfusion CT for the assessment of tumour vascularity: which protocol? Br J Radiol. 2003;76 Spec No 1:S36-S42.

40. Miles KA. Functional computed tomography in oncology. Eur J Cancer. 2002;38(16):2079-2084

41. Materne R, van Beers BE, Smith AM, et al. Non-invasive quantification of liver perfusion with dynamic computed tomography and a dual-input one-compartmental model. Clin Sci (Lond). 2000;99(6):517-525.

42. Cuenod C, Leconte I, Siauve N, et al. Early changes in liver perfusion caused by occult metastases in rats: detection with quantitative CT. Radiology. 2001;218(2):556-561.

43. Johnson JA, Wilson TA. A model for capillary exchange. Am J Physiol 1966;210(6):1299-1303.

44. Wang L, van den Bos IC, Hussain SM, et al. Post-processing of dynamic gadolinium-enhanced magnetic resonance imaging exams of the liver: explanation and potential clinical applications for color-coded qualitative and quantitative analysis. Acta Radiol. 2008;49(1):6-18.

45. Laking GR, West C, Buckley DL, Matthews J, Price PM. Imaging vascular physiology to monitor cancer treatment. Crit Rev Oncol Hematol. 2006;58(2):95-113.

46. Hashimoto K, Murakami T, Dono K, et al. Quantitative tissue blood flow measurement of the liver parenchyma: comparison between xenon CT and perfusion CT. Dig Dis Sci. 2007;52(4):943-949.

47. van den Bos IC, Hussain SM, de Man RA, et al. Primary hepatocellular lesions: imaging findings on state-of-the-art magnetic resonance imaging, with pathologic correlation. Curr Probl Diagn Radiol. 2008; 37(3):104-114.

48. Hussain SM, van den Bos IC, Dwarkasing RS, Kuiper JW, den Hollander J. Hepatocellular adenoma: findings at state-of-the-art magnetic resonance imaging, ultrasound, computed tomography and pathologic analysis. Eur Radiol. 2006;16(9):1873-1886.

49. Hussain SM, Semelka RC, Mitchell DG. MR imaging of hepatocellular carcinoma. Magn Reson Imaging Clin N Am. 2002;10(1):31-52,

50. Ippolito D, Sironi S, Pozzi M, et al. Hepatocellular carcinoma in cirrhotic liver disease: functional computed tomography with perfusion imaging in the assessment of tumor vascularization. Acad Radiol. 2008; 15(7):919-927.

51. Meijerink MR, van Waesberghe JH, van der Weide L, et al. Total-livervolume perfusion CT using 3-D image fusion to improve detection and characterization of liver metastases. Eur Radiol. 2008;18(10): 2345-2354.

52. Miles KA, Leggett DA, Kelley BB, et al. In vivo assessment of neovascularization of liver metastases using perfusion $\mathrm{CT}$. $\mathrm{Br} J$ Radiol. 1998;71(843):276-281.

53. Lupescu IG, Grasu M, Capsa R, Pitrop A, Georgescu SA. Hepatic perfusion disorders: computer-tomographic and magnetic resonance imaging. J Gastrointestin Liver Dis. 2006;15(3):273-279.

54. Faria SC, Ng CS, Hess KR, et al. CT quantification of effects of thalidomide in patients with metastatic renal cell carcinoma. AJR Am J Roentgenol. 2007;189(2):378-385.

55. Leggett DA, Kelley BB, Bunce IH, Miles KA. Colorectal cancer: diagnostic potential of CT measurements of hepatic perfusion and implications for contrast enhancement protocols. Radiology. 1997;205(3): 716-720.

56. Cuenod CA, Fournier L, Balvay D, Guinebretiere JM. Tumor angiogenesis: pathophysiology and implications for contrast-enhanced MRI and CT assessment. Abdom Imaging. 2006;31(2):188-193.

57. Luca A, Caruso S, Milazzo M, et al. Multidetector-row computed tomography (MDCT) for the diagnosis of hepatocellular carcinoma in cirrhotic candidates for liver transplantation: prevalence of radiological vascular patterns and histological correlation with liver explants. Eur Radiol. 2010;20(4):898-907.
58. Hussain SM, Semelka RC. Liver masses. Magn Reson Imaging Clin $N$ Am. 2005;13(2):255-275.

59. Boll DT, Merkle EM. Diffuse liver disease: strategies for hepatic CT and MR imaging. Radiographics. 2009;29(6):1591-1614.

60. Weidekamm C, Cejna M, Kramer L, Peck-Radosavljevic M, Bader TR. Effects of TIPS on liver perfusion measured by dynamic CT. Am J Roentgenol. 2005;184(2):505-510.

61. van Beers BE, Leconte I, Materne R, et al. Hepatic perfusion parameters in chronic liver disease: dynamic CT measurements correlated with disease severity. Am J Roentgenol. 2001;176(3):667-673.

62. Hashimoto K, Murakami T, Dono K, et al. Assessment of the severity of liver disease and fibrotic change: the usefulness of hepatic $\mathrm{CT}$ perfusion imaging. Oncol Rep. 2006;16(4):677-683.

63. Zhou XP, Lu T, Wei YG, Chen XZ. Liver volume variation in patients with virus-induced cirrhosis: findings on MDCT. AJR Am J Roentgenol. 2007;189(3):W153-W159.

64. Tu R, Xia LP, Yu AL, Wu L. Assessment of hepatic functional reserve by cirrhosis grading and liver volume measurement using CT. World $J$ Gastroenterol. 2007;13(29):3956-3961.

65. Gryspeerdt S, van Hoe L, Marchal G, Baert AL. Evaluation of hepatic perfusion disorders with double-phase spiral CT. Radiographics. 1997;17(2):337-348.

66. Edge SB, Byrd DR, Carducci MA, Compton CC, editors. AJCC Cancer Staging Handbook. 7th ed. New York: Springer-Verlag; 2010.

67. Zhu AX, Holalkere NS, Muzikansky A, Horgan K, Sahani DV. Early antiangiogenic activity of bevacizumab evaluated by computed tomography perfusion scan in patients with advanced hepatocellular carcinoma. Oncologist. 2008;13(2):120-125.

68. Gwyther SJ, Schwartz LH. How to assess anti-tumour efficacy by imaging techniques. Eur J Cancer. 2008;44(1):39-45.

69. Husband JE, Schwartz LH, Spencer J, et al. Evaluation of the response to treatment of solid tumours - a consensus statement of the International Cancer Imaging Society. $\mathrm{Br} J$ Cancer. 2004;90(12):2256-2260.

70. Schima W, Ba-Ssalamah A, Kurtaran A, Schindl M, Gruenberger T. Post-treatment imaging of liver tumours. Cancer Imaging. 2007;7 Spec No A:S28-S36.

71. Jain RK. Normalization of tumor vasculature: an emerging concept in antiangiogenic therapy. Science. 2005;307(5706):58-62.

72. World Health Organization. WHO Handbook for Reporting Results of Cancer Treatment. WHO Publication No. 48. Geneva: WHO; 1979.

73. Miller AB, Hoogstraten B, Staquet M, Winkler A. Reporting results of cancer treatment. Cancer. 1981;47(1):207-214.

74. Therasse P, Arbuck SG, Eisenhauer EA, et al. New guidelines to evaluate the response to treatment in solid tumors. European Organization for Research and Treatment of Cancer, National Cancer Institute of the United States, National Cancer Institute of Canada. J Natl Cancer Inst. 2000;92(3):205-216

75. Eisenhauer EA, Therasse P, Bogaerts J, et al. New response evaluation criteria in solid tumours: revised RECIST guideline (version 1.1). Eur J Cancer. 2009;45(2):228-247.

76. Therasse P, Eisenhauer EA, Verweij J. RECIST revisited: a review of validation studies on tumour assessment. Eur J Cancer 2006;42(8): 1031-1039.

77. van Persijn van Meerten EL, Gelderblom H, Bloem JL. RECIST revised: implications for the radiologist. A review article on the modified RECIST guideline. Eur Radiol. 2010;20(6):1456-1467.

78. Kawamoto S, Permpongkosol S, Bluemke DA, Fishman EK, Solomon SB. Sequential changes after radiofrequency ablation and cryoablation of renal neoplasms: role of CT and MR imaging. Radiographics. 2007;27(2):343-355.

79. Rosen MA. Use of modified RECIST criteria to improve response assessment in targeted therapies: challenges and opportunities. Cancer Biol Ther. 2010;9(1):15-19.

80. Forner A, Ayuso C, Varela M, et al. Evaluation of tumor response after locoregional therapies in hepatocellular carcinoma: are response evaluation criteria in solid tumors reliable? Cancer. 2009;115(3):616-623. 
81. Llovet JM, Di Bisceglie AM, Bruix J, et al. Design and endpoints of clinical trials in hepatocellular carcinoma. J Natl Cancer Inst. 2008; 100(10):698-711.

82. Zhu AX, Blaszkowsky LS, Ryan DP, et al. Phase II study of gemcitabine and oxaliplatin in combination with bevacizumab in patients with advanced hepatocellular carcinoma. J Clin Oncol. 2006;24(12): 1898-1903.

83. Wang B, Gao ZQ, Yan X. Correlative study of angiogenesis and dynamic contrast-enhanced magnetic resonance imaging features of hepatocellular carcinoma. Acta Radiol. 2005;46(4):353-358.

84. Mabille M, Vanel D, Albiter M, et al. Follow-up of hepatic and peritoneal metastases of gastrointestinal tumors (GIST) under Imatinib therapy requires different criteria of radiological evaluation (size is not everything!!!). Eur J Radiol. 2009;69(2):204-208.

85. Bruix J, Sherman M, Llovet JM, et al. Clinical management of hepatocellular carcinoma. Conclusions of the Barcelona-2000 EASL conference. European Association for the Study of the Liver. J Hepatol. 2001;35(3):421-430

86. White RR, Schwartz LH, Munoz JA, et al. Assessing the optimal duration of chemotherapy in patients with colorectal liver metastases. J Surg Oncol. 2008;97(7):601-604.

87. Abou-Alfa GK, Schwartz L, Ricci S, et al. Phase II study of sorafenib in patients with advanced hepatocellular carcinoma. J Clin Oncol. 2006;24(26):4293-300.

88. Wang L, Abou-Alfa GK, Saltz LB, et al. Novel methodology of response assessment in hepatocellular carcinoma (HCC) - assessing response by change in tumor enhancement in distinction from conventional means J Clin Oncol. 2004;24(22):3107.

89. Chi KH, Liao CS, Chang CC, et al. Angiogenic blockade and radiotherapy in hepatocellular carcinoma. Int J Radiat Oncol Biol Phys. Epub 2010 Feb 2.

90. Wang L, Schwartz LH, Mazumdar M, et al. Impact of measurement technique on response assessment criteria in colorectal cancer J Clin Oncol. 2003;22:278.

91. Xiong HQ, Herbst R, Faria SC, et al. A phase I surrogate endpoint study of SU6668 in patients with solid tumors. Invest New Drugs. 2004;22(4):459-466.

92. Thomas AL, Morgan B, Horsfield MA, et al. Phase I study of the safety, tolerability, pharmacokinetics, and pharmacodynamics of PTK787/ ZK 222584 administered twice daily in patients with advanced cancer. J Clin Oncol. 2005;23(18):4162-4171.

93. Kan Z, Phongkitkarun S, Kobayashi S, et al. Functional CT for quantifying tumor perfusion in antiangiogenic therapy in a rat model. Radiology. 2005;237(1):151-158.

94. Chen WX, Min PQ, Song B, Xiao BL, Liu Y, Ge YH. Single-level dynamic spiral CT of hepatocellular carcinoma: correlation between imaging features and density of tumor microvessels. World $J$ Gastroenterol. 2004;10(1):67-72.

95. Kan Z, Kobayashi S, Phongkitkarun S, Charnsangavej C. Functional CT quantification of tumor perfusion after transhepatic arterial embolization in a rat model. Radiology. 2005;237(1):144-150.

96. Vauthey JN, Chaoui A, Do KA, et al. Standardized measurement of the future liver remnant prior to extended liver resection: methodology and clinical associations. Surgery. 2000;127(5):512-519.

97. Soyer P, Roche A, Elias D, Levesque M. Hepatic metastases from colorectal cancer: influence of hepatic volumetric analysis on surgical decision making. Radiology. 1992;184(3):695-697.

98. Wakabayashi H, Nakano S, Ishimura K, et al. Changes in arterial and portal perfusion in embolized and nonembolized hepatic lobes after portal vein embolization evaluated by helical computed tomography. Surg Today. 2001;31(11):991-995.

99. Stockmann M, Lock JF, Riecke B, et al. Prediction of postoperative outcome after hepatectomy with a new bedside test for maximal liver function capacity. Ann Surg. 2009;250(1):119-125.

100. Zappa M, Dondero F, Sibert A, et al. Liver regeneration at day 7 after right hepatectomy: global and segmental volumetric analysis by using CT. Radiology. 2009;252(2):426-432.
101. Kayashima H, Taketomi A, Yonemura Y, et al. Accuracy of an ageadjusted formula in assessing the graft volume in living donor liver transplantation. Liver Transpl. 2008;14(9):1366-1371.

102. Szijarto A, Hargitai B, Fischer S, Darvas K, Kupcsulik P. Two-staged procedure of portal ligation and hepatectomy monitored by ICG clearance. J Invest Surg. 2009;22(1):63-68.

103. Sirichindakul B, Nonthasoot B, Taesombat W, et al. Role of portal vein embolization in hepatobiliary malignancy. Hepatogastroenterology. 2007;54(80):2297-2300.

104. Ferrero A, Vigano L, Polastri R, et al. Postoperative liver dysfunction and future remnant liver: where is the limit? Results of a prospective study. World J Surg. 2007;31(8):1643-1651.

105. Uslu Tutar N, Kirbas I, Ozturk A, et al. Computed tomography volumetric follow-up of graft volume in living related liver recipients. Transplant Proc. 2007;39(4):1175-1177.

106. Mitsumori A, Nagaya I, Kimoto S, et al. Preoperative evaluation of hepatic functional reserve following hepatectomy by technetium-99m galactosyl human serum albumin liver scintigraphy and computed tomography. Eur J Nucl Med. 1998;25(10):1377-1382.

107. de Graaf W, van Lienden KP, van Gulik TM, Bennink RJ. (99m) Tc-mebrofenin hepatobiliary scintigraphy with SPECT for the assessment of hepatic function and liver functional volume before partial hepatectomy. J Nucl Med. 2010;51(2):229-236.

108. Muller SA, Blauer K, Kremer M, et al. Exact CT-based liver volume calculation including nonmetabolic liver tissue in three-dimensional liver reconstruction. J Surg Res. 2010;160(2):236-243.

109. Dello SA, van Dam RM, Slangen JJ, et al. Liver volumetry plug and play: do it yourself with ImageJ. World J Surg. 2007;31(11):2215-2221.

110. Das M, Sauer F, Schoepf UJ, et al. Augmented reality visualization for CT-guided interventions: system description, feasibility, and initial evaluation in an abdominal phantom. Radiology. 2006;240(1):230-235.

111. Ricke J, Wust P, Wieners G, et al. Liver malignancies: CT-guided interstitial brachytherapy in patients with unfavorable lesions for thermal ablation. J Vasc Interv Radiol. 2004;15(11):1279-1286.

112. Mohnike K, Wieners G, Schwartz F, Seidensticker M, Pech M, Ruehl R, et al. Computed tomography-guided high-dose-rate brachytherapy in hepatocellular carcinoma: safety, efficacy, and effect on survival. Int $J$ Radiat Oncol Biol Phys. Epub 2010 Jan 5.

113. Ruhl R, Seidensticker M, Peters N, et al. Hepatocellular carcinoma and liver cirrhosis: assessment of the liver function after Yttrium-90 radioembolization with resin microspheres or after CT-guided highdose-rate brachytherapy. Dig Dis. 2009;27(2):189-199.

114. Goyal N, Jain N, Rachapalli V, Cochlin DL, Robinson M. Non-invasive evaluation of liver cirrhosis using ultrasound. Clin Radiol. 2009; 64(11):1056-1066.

115. Chen S, Urban MW, Pislaru C, Kinnick R, Greenleaf JF. Liver elasticity and viscosity quantification using shearwave dispersion ultrasound vibrometry (SDUV). Conf Proc IEEE Eng Med Biol Soc. 2009;2009:2252-2255.

116. Tchelepi H, Ralls PW. Ultrasound of focal liver masses. Ultrasound $Q$. 2004;20(4):155-169.

117. Castera L, Forns X, Alberti A. Non-invasive evaluation of liver fibrosis using transient elastography. J Hepatol. 2008;48(5):835-847.

118. Talwalkar JA, Kurtz DM, Schoenleber SJ, West CP, Montori VM. Ultrasound-based transient elastography for the detection of hepatic fibrosis: systematic review and meta-analysis. Clin Gastroenterol Hepatol. 2007;5(10):1214-1220.

119. Han KH, Yoon KT. New diagnostic method for liver fibrosis and cirrhosis. Intervirology. 2008;51 Suppl 1:11-16.

120. Goh V, Padhani AR. Imaging tumor angiogenesis: functional assessment using MDCT or MRI? Abdom Imaging. 2006;31(2):194-199.

121. Kim YK, Kim CS, Chung GH, et al. Comparison of gadobenate dimeglumine-enhanced dynamic MRI and 16-MDCT for the detection of hepatocellular carcinoma. AJR Am J Roentgenol. 2006; 186(1):149-157.

122. Gossuin Y, Gillis P, Hocq A, Vuong QL, Roch A. Magnetic resonance relaxation properties of superparamagnetic particles. Wiley Interdiscip Rev Nanomed Nanobiotechnol. 2009;1(3):299-310. 
123. Lupescu IG, Capsa RA, Gheorghe L, Herlea V, Georgescu SA. Tissue specific MR contrast media role in the differential diagnosis of cirrhotic liver nodules. J Gastrointestin Liver Dis. 2008;17(3):341-346.

124. Zech CJ, Herrmann KA, Reiser MF, Schoenberg SO. MR imaging in patients with suspected liver metastases: value of liver-specific contrast agent Gd-EOB-DTPA. Magn Reson Med Sci. 2007;6(1):43-52.

125. Galbraith SM, Lodge MA, Taylor NJ, et al. Reproducibility of dynamic contrast-enhanced MRI in human muscle and tumours: comparison of quantitative and semi-quantitative analysis. NMR Biomed. 2002 15(2):132-142.

126. Le Bihan D. Molecular diffusion nuclear magnetic resonance imaging. Magn Reson Q. 1991;7(1):1-30.

127. Koh DM, Collins DJ. Diffusion-weighted MRI in the body: applications and challenges in oncology. AJR Am J Roentgenol. 2007;188(6) $1622-1635$

128. Parikh T, Drew SJ, Lee VS, et al. Focal liver lesion detection and characterization with diffusion-weighted MR imaging: comparison with standard breath-hold T2-weighted imaging. Radiology. 2008; 246(3):812-822.

129. Taouli B, Martin AJ, Qayyum A, et al. Parallel imaging and diffusion tensor imaging for diffusion-weighted MRI of the liver: preliminary experience in healthy volunteers. AJR Am J Roentgenol. 2004;183(3): 677-680.

130. Taouli B, Chouli M, Martin AJ, et al. Chronic hepatitis: role of diffusionweighted imaging and diffusion tensor imaging for the diagnosis of liver fibrosis and inflammation. J Magn Reson Imaging. 2008;28(1):89-95.

131. Mannelli L, Kim S, Hajdu CH, et al. Assessment of tumor necrosis of hepatocellular carcinoma after chemoembolization: diffusion-weighted and contrast-enhanced MRI with histopathologic correlation of the explanted liver. AJR Am J Roentgenol. 2009;193(4):1044-1052.

132. Hardie AD, Naik M, Hecht EM, et al. Diagnosis of liver metastases: value of diffusion-weighted MRI compared with gadolinium-enhanced MRI. Eur Radiol. 2010;20(6):1431-1441.

133. Nishie A, Tajima T, Ishigami K, et al. Detection of hepatocellular carcinoma (HCC) using super paramagnetic iron oxide (SPIO)-enhanced MRI: added value of diffusion-weighted imaging (DWI). J Magn Reson Imaging. 2010;31(2):373-382.

134. Catalano OA, Choy G, Zhu A, Hahn PF, Sahani DV. Differentiation of malignant thrombus from bland thrombus of the portal vein in patients with hepatocellular carcinoma: application of diffusion-weighted MR imaging. Radiology. 2010;254(1):154-162.

135. Taouli B, Koh DM. Diffusion-weighted MR imaging of the liver Radiology. 2010;254(1):47-66.

136. Colagrande S, Pasquinelli F, Mazzoni LN, Belli G, Virgili G. MRdiffusion weighted imaging of healthy liver parenchyma: repeatability and reproducibility of apparent diffusion coefficient measurement. J Magn Reson Imaging. 2010;31(4):912-920.

137. Dagnelie PC, Leij-Halfwerk S. Magnetic resonance spectroscopy to study hepatic metabolism in diffuse liver diseases, diabetes and cancer World J Gastroenterol. 2010;16(13):1577-1586.

138. Noren B, Dahlqvist O, Lundberg P, et al. Separation of advanced from mild fibrosis in diffuse liver disease using 31P magnetic resonance spectroscopy. Eur J Radiol. 2008;66(2):313-320.

139. Dagnelie PC, Sijens PE, Kraus DJ, Planting AS, van Dijk P. Abnormal liver metabolism in cancer patients detected by (31)P MR spectroscopy. NMR Biomed. 1999;12(8):535-544.

140. Qayyum A. MR spectroscopy of the liver: principles and clinical applications. Radiographics. 2009;29(6):1653-1664.

141. Pineda N, Sharma P, Xu Q, et al. Measurement of hepatic lipid: highspeed T2-corrected multiecho acquisition at 1H MR spectroscopy - a rapid and accurate technique. Radiology. 2009;252(2):568-576.

142. Sha W, da Costa KA, Fischer LM, et al. Metabolomic profiling can predict which humans will develop liver dysfunction when deprived of dietary choline. FASEB J. Epub 2010 Apr 6.

143. Fischbach F, Schirmer T, Thormann M, et al. Quantitative proton magnetic resonance spectroscopy of the normal liver and malignant hepatic lesions at 3.0 Tesla. Eur Radiol. 2008;18(11):2549-2558.
144. Hu S, Lustig M, Balakrishnan A, et al. 3D compressed sensing for highly accelerated hyperpolarized (13)C MRSI with in vivo applications to transgenic mouse models of cancer. Magn Reson Med. 2010;63(2):312-321.

145. Chmelik M, Schmid AI, Gruber S, et al. Three-dimensional highresolution magnetic resonance spectroscopic imaging for absolute quantification of 31P metabolites in human liver. Magn Reson Med. 2008;60(4):796-802.

146. Huwart L, van Beers BE. MR elastography. Gastroenterol Clin Biol. 2008;32(6 Suppl 1):68-72.

147. Watanabe H, Kanematsu M, Kitagawa T, et al. MR elastography of the liver at $3 \mathrm{~T}$ with cine-tagging and bending energy analysis: preliminary results. Eur Radiol. Epub 2010 May 4.

148. Salameh N, Larrat B, Abarca-Quinones J, et al. Early detection of steatohepatitis in fatty rat liver by using MR elastography. Radiology. 2009;253(1):90-97.

149. Asbach P, Klatt D, Hamhaber U, et al. Assessment of liver viscoelasticity using multifrequency MR elastography. Magn Reson Med. 2008; 60(2):373-379.

150. de Graaf W, Bennink RJ, Vetelainen R, van Gulik TM. Nuclear imaging techniques for the assessment of hepatic function in liver surgery and transplantation. J Nucl Med. 2010;51(5):742-752.

151. Nanashima A, Yamaguchi H, Shibasaki S, et al. Relationship between CT volumetry and functional liver volume using technetium-99m galactosyl serum albumin scintigraphy in patients undergoing preoperative portal vein embolization before major hepatectomy: a preliminary study. Dig Dis Sci. 2006;51(7):1190-1195.

152. Dinant S, de Graaf W, Verwer BJ, et al. Risk assessment of posthepatectomy liver failure using hepatobiliary scintigraphy and CT volumetry. J Nucl Med. 2007;48(5):685-692.

153. Kawamura E, Shiomi S, Ishizu H, et al. Natural course of changes in hepatic functional reserve in patients with chronic liver diseases evaluated by scintigraphy with GSA. Hepatol Res. 2003;27(2):129-135.

154. Onodera Y, Takahashi K, Togashi T. Clinical assessment of hepatic functional reserve using ${ }^{99 \mathrm{~m}}$ Tc DTPA galactosyl human serum albumin SPECT to prognosticate chronic hepatic diseases - validation of the use of SPECT and a new indicator. Ann Nucl Med. 2003;17(3):181-188.

155. Sasaki N, Shiomi S, Iwata Y, et al. Clinical usefulness of scintigraphy with 99mTc-galactosyl-human serum albumin for prognosis of cirrhosis of the liver. J Nucl Med. 1999;40(10):1652-1656.

156. Kira T, Tomiguchi S, Takahashi M, et al. Correlation of $99 \mathrm{mTc}-\mathrm{GSA}$ hepatic scintigraphy with liver biopsies in patients with chronic active hepatitis type C. Radiat Med. 1999;17(2):125-130.

157. Shuke N, Aburano T, Okizaki A, et al. Estimation of fractional liver uptake and blood retention of 99mTc-DTPA-galactosyl human serum albumin: an application of a simple graphical method to dynamic SPECT. Nucl Med Commun. 2003;24(5):503-511.

158. Nishiyama Y, Yamamoto Y, Hino I, et al. ${ }^{99 m}$ Tc galactosyl human serum albumin liver dynamic SPET for pre-operative assessment of hepatectomy in relation to percutaneous transhepatic portal embolization. Nucl Med Commun. 2003;24(7):809-817.

159. Zhao B, Schwartz LH, Larson SM. Imaging surrogates of tumor response to therapy: anatomic and functional biomarkers. J Nucl Med. 2009;50(2):239-249.

160. Flower MA, Zweit J, Hall AD, et al. 62Cu-PTSM and PET used for the assessment of angiotensin II-induced blood flow changes in patients with colorectal liver metastases. Eur J Nucl Med. 2001;28(1): 99-103.

161. He YX, Guo QY. Clinical applications and advances of positron emission tomography with fluorine-18-fluorodeoxyglucose (18F-FDG) in the diagnosis of liver neoplasms. Postgrad Med J 2008;84(991):246-251.

162. Wiering B, Vogel WV, Ruers TJ, Oyen WJ. Controversies in the management of colorectal liver metastases: role of PET and PET/CT. Dig Surg. 2008;25(6):413-420. 
163. Daldrup-Link HE, Simon GH, Brasch RC. Imaging of tumor angiogenesis: current approaches and future prospects. Curr Pharm Des. 2006;12(21):2661-2672.

164. Choyke PL, Dwyer AJ, Knopp MV. Functional tumor imaging with dynamic contrast-enhanced magnetic resonance imaging. J Magn Reson Imaging. 2003;17(5):509-520.

165. Bolus NE, George R, Washington J, Newcomer BR. PET/MRI: the blended-modality choice of the future? J Nucl Med Technol. 2009;37(2):63-71; quiz 72-73.

166. Hicks RJ, Lau EW. PET/MRI: a different spin from under the rim. Eur J Nucl Med Mol Imaging. 2009;36(0Suppl 1):S10-S14.

167. Judenhofer MS, Wehrl HF, Newport DF, et al. Simultaneous PETMRI: a new approach for functional and morphological imaging. Nat Med. 2008;14(4):459-465.

168. Wehrl HF, Judenhofer MS, Wiehr S, Pichler BJ. Pre-clinical PET/MR: technological advances and new perspectives in biomedical research. Eur J Nucl Med Mol Imaging. 2009;36(Suppl 1):S56-S68.

169. Glaus C, Rossin R, Welch MJ, Bao G. In vivo evaluation of (64)Culabeled magnetic nanoparticles as a dual-modality PET/MR imaging agent. Bioconjug Chem. 2010;21(4):715-722.
170. Pichler BJ, Judenhofer MS, Pfannenberg C. Multimodal imaging approaches: PET/CT and PET/MRI. Handb Exp Pharmacol. 2008. (185 Pt 1):109-132.

171. Gaa J, Rummeny EJ, Seemann MD. Whole-body imaging with PET/MRI. Eur J Med Res. 2004;9(6):309-312.

172. Pichler BJ, Kolb A, Nagele T, Schlemmer HP. PET/MRI: paving the way for the next generation of clinical multimodality imaging applications. J Nucl Med. 2010;51(3):333-336.

173. Cui Y, Tan Y, Zhao B, et al. Malignant lesion segmentation in contrast-enhanced breast MR images based on the marker-controlled watershed. Med Phys. 2009;36(10):4359-4369.

174. Wahl RL, Jacene H, Kasamon Y, Lodge MA. From RECIST to PERCIST: evolving considerations for PET response criteria in solid tumors. J Nucl Med. 2009;50 Suppl 1:122S-150S.

\section{Publish your work in this journal}

Hepatic Medicine: Evidence and Research is an international, peerreviewed, open access journal covering all aspects of adult and pediatric hepatology in the clinic and laboratory including the following topics: Pathology, pathophysiology of hepatic disease; Investigation and treatment of hepatic disease; Pharmacology of drugs used for the treatment of hepatic disease. Issues of patient safety and quality of care will also be considered. The manuscript management system is completely online and includes a very quick and fair peer-review system, which is all easy to use. Visit http://www.dovepress.com/ testimonials.php to read real quotes from published authors.

Submit your manuscript here: http://www.dovepress.com/hepatic-medicine-evidence-and-research-journal 